A. Brömme, C. Busch, A. Dantcheva, C. Rathgeb and A. Uhl (Eds.): BIOSIG 2018, Lecture Notes in Informatics (LNI), Gesellschaft für Informatik, Bonn $2018 \quad 1$

\title{
Robust Clustering-based Segmentation Methods for Fingerprint Recognition ${ }^{1}$
}

\author{
Pedro M. Ferreira ${ }^{2,4}$, Ana F. Sequeira ${ }^{3}$, Jaime S. Cardoso ${ }^{2,4}$, Ana Rebelo ${ }^{2,5}$
}

\begin{abstract}
Fingerprint recognition has been widely studied for more than 45 years and yet it remains an intriguing pattern recognition problem. This paper focuses on the foreground mask estimation which is crucial for the accuracy of a fingerprint recognition system. The method consists of a robust cluster-based fingerprint segmentation framework incorporating an additional step to deal with pixels that were rejected as foreground in a decision considered not reliable enough. These rejected pixels are then further analysed for a more accurate classification. The procedure falls in the paradigm of classification with reject option - a viable option in several real world applications of machine learning and pattern recognition, where the cost of misclassifying observations is high. The present work expands a previous method based on the fuzzy C-means clustering with two variations regarding: i) the filters used; and ii) the clustering method for pixel classification as foreground/background. Experimental results demonstrate improved results on FVC datasets comparing with state-of-the-art methods even including methodologies based on deep learning architectures.
\end{abstract}

Keywords: Biometric recognition, fingerprint, segmentation, clustering, morphological operations.

\section{Introduction}

Fingerprint recognition systems have been used in a wide range of personal identification domains, such as civil identification, access control and forensics. Although very effective solutions are currently available, it cannot be considered a fully solved problem, and the design of accurate, interoperable, and computationally light algorithms is still an open issue [Ma09]. A fingerprint recognition system is composed by several processing steps, usually starting with a fingerprint foreground mask estimation (FME), passing by the enhancement and binarization steps ending with minutiae extraction and matching [Ma09] . This paper focuses on one of the first steps: foreground mask estimation, which is crucial for the accuracy of the system as a well done fingerprint segmentation promotes both the elimination of spurious minutiae close to the foreground boundaries and the reduction of the computation time of the following steps.

\footnotetext{
${ }^{2}$ INESC TEC, Porto, Portugal, Email: \{pmmferreira,jsc,arebelo\}@ @inesctec.pt

${ }^{3}$ IrisGuard, Research \& Development, Milton Keynes, United Kingdom, Email: asequeira @ irisguard.com

${ }^{4}$ Faculdade de Engenharia, Porto, Portugal

${ }^{5}$ UPT, Porto, Portugal

${ }^{1}$ This work was funded by the Protect "NanoSTIMA: Macro-to-Nano Human Sensing: Towards Integrated Multimodal Health Monitoring and Analytics/NORTE-010145-FEDER000016" financed by the North Portugal Regional Operational Programme (NORTE 2020), under PORTUGAL 2020 Partnership Agreement, and through the European Regional Development FUND (ERDF), and also by Fundação para a Ciência e a Tecnologia (FCT) within PhD and BPD grants with numbers SFRH/BD/102177/2014 and SFRH/BPD/101439/2014.
} 
This paper expands a previous work [FSR15] where Ferreira et al. followed the morphological fingerprint segmentation algorithm presented by Fahmy et al. [FT13]. In the present work, a more general framework of the method is presented where the method is instantiated by the combination of three different filters: range, entropy and variance filters; and three clustering methods: fuzzy c-means (FCM), k-means and gaussian mixture models (GMM) for pixel classification. Experimental results demonstrate significant progress on the four existing FVC datasets: FVC2000, FVC2002, FVC2004 and FVC2006. The main contributions of this paper are: i) the general framwork incorporating different combinations of filters and clustering methods; ii) the additional third cluster for the unreliably classified pixels applying the conceptual idea of reject option; iii) the comparison with state-of-the-art methods, including a deep learning approach, with leading results being obtained by the proposed method.

The evaluation was made using masks manually delineated and carried out using state-ofthe-art segmentation methods. Additionally, an indirect evaluation involving the integration of the proposed modules in a complete fingerprint recognition system and measuring the gains obtained was made. Using an in-house robust fingerprint verification $(R F V)$ system and replacing the correspondent modules with the proposed methods and state-of-theart segmentation methods.

This paper is organized as follows: section 2 contains the related work; section 3 present the proposed method; in section 4 are presented the frameworks used for evaluation and the experimental results obtained for fingerprint mask estimation are discussed; and in section 5 the work is concluded and future work is discussed.

\section{Related Work}

Fingerprint segmentation methods known from literature can be roughly divided into blockwise methods and pixel-wise methods [NM14]. Pixel-wise methods [BG01, WTG07, FT13] classify pixels through the analysis of pixel-wise features. Block-wise methods [Ch04, Li11] first partition a fingerprint image into non overlapping blocks of the same size, and then classify the blocks into foreground and background based on extracted block-wise features. The commonly used features in fingerprint segmentation include gray-level features, orientation features, frequency domain features among others [NM14].

Bazen et al. [BG01] suggested a pixel-wise method, in which three features (coherence, mean and variance) are computed for each pixel and then a linear classifier associates the pixel with the foreground or the background. The method presented by Chen et al. [Ch04] uses a block cluster degree along with a linear classifier. Wu et al. [WTG07] proposed a fingerprint segmentation method based on the Harris corner detector. The image pixels with the strongest Harris response are considered as foreground pixels and the final segmentation is obtained as their convex hull. Liu et al. [Li11] suggested a classification using the AdaBoost classifier with two novel features (block entropy and block gradient entropy) and several commonly used features (coherence, mean, variance and Gabor features). Kang et al. [KZ09] proposed a method based on fuzzy c-means by modifying the 
objective function in the Szilagyis algorithm via introducing histogram-based weighting. This method is evaluated in a test image in original form and also corrupted by gaussian and salt and pepper noise. Another method based in an adaptation of fuzzy c-means is proposed by Raghotham et al. [Ra12]. This method combines the probabilistic fuzzy cmeans clustering (PFCM) algorithm (which is a mix of probabilistic c-means clustering (PCM) and fuzzy c-means clustering (FCM) algorithm allowing to overcome the noise sensitivity defect in FCM and coincident cluster problem in PCM) with an adaptative level set method. The evaluation of the method is done by visual inspection of some examples. Fahmy et al. [FT13] proposed a fingerprint segmentation method based on morphological operations. A range filter is applied to the fingerprint image followed by an adaptative thresholding binarization. A final morphological post-processing step is performed in order to remove holes in both foreground and background.

Recent works propose to learn a Convolutional Neural Network (CNN) to distinguish between background and foreground of a fingerprint [NCKJ18, Ta17].

\section{Proposed method for Robust Fingerprint Segmentation}

Ferreira et al. [FSR15] proposed a robust fingerprint segmentation algorithm, in which the range feature is extracted from the image and clustered using a Fuzzy C-means (FCM) approach. Fingerprint images are frequently contaminated by noise and the method presented was capable of coping with it due to the association of clustering and FCM algorithm.

The present work expands the previous study by reinforcing the proposal of a robust cluster-based fingerprint segmentation framework able to deal with the sensor interoperability problem. The high-level operations that compose the proposed fingerprint segmentation framework are presented in Figure 1. By combining 3 different features (range, entropy and variance) and 3 clustering algorithms (FCM, $k$-means and gaussian mixture models (GMM)) a total of 9 combinations are evaluated in the proposed segmentation framework. In Figure 2 is depicted one example of the output images in each step.

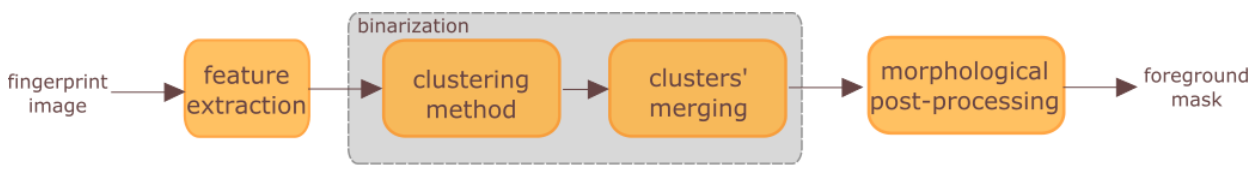

Fig. 1: Architecture of the fingerprint segmentation method proposed.

Feature extraction is the first step. The extracted features should be reliable enough to represent the foreground of fingerprint images captured with different sensor types.

Clustering technique is applied to the extracted features followed by a robust cluster' merging procedure. Although fingerprint segmentation is commonly addressed as a twoclass clustering task, in which the goal is to distinguish the foreground cluster from the background one, here the data is clustered into three clusters. This procedure can be viewed in the context of classification with reject option [SC10, So14, CKB15] which is a viable option in several real world applications of machine learning and pattern recognition, where the cost of misclassifying observations is high (e.g., as it happens in computer aided 
diagnosis systems). It consists on withholding the automatic classification of an observation, if the decision is considered not sufficiently reliable. Then, the rejected observation is typically handled by a different classifier. In our case, all pixels of the third cluster can be seen as rejected observations, which are further analysed in a more accurate decision process. That is, a cluster' merging procedure, based on neighborhood- and variance-based criteria, is applied to classify the rejected pixels into foreground or background.

Morphological Post-processing is the final step of the method. At this stage, the binary foreground mask is composed by several disjoint binary regions with ragged boundaries. Therefore, a post-processing is required to obtain the final foreground mask. The postprocessing operations include: 1) a morphological closing filter to join small adjacent regions; 2) a region filling algorithm to fill interior holes of the binary objects; 3) a morphological opening filter to eliminate thin protrusions and, generally, smooth the contours; and 4) the largest binary component of the image is selected and assumed as the final foreground mask. One example of output of this phase is illustrated in Figure 2(e).

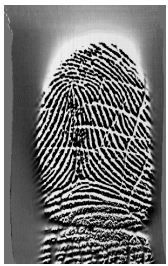

(a)

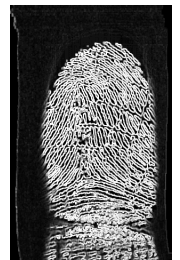

(b)

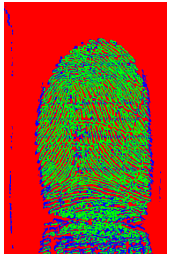

(c)

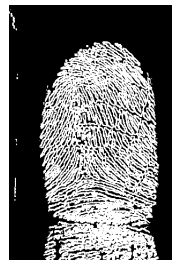

(d)

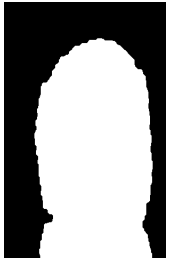

(e)

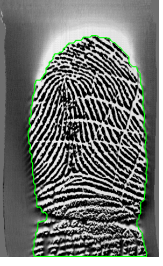

(f)

Fig. 2: Outputs of the proposed fingerprint segmentation framework (RANGE feature and FCM method): (a) Original image, (b) Range feature, (c) FCM clustering for $C=3$, (d) Binary image, (e) Final foreground mask, and (f) Foreground boundary superimposed on the original image.

\section{Experimental results and discussion of the Segmentation Methods}

A total of 9 combinations of 3 extracted features and 3 clustering algorithms were tested and compared against four state-of-the-art segmentation methods, namely: 1) the baseline segmentation algorithm [FT13]; 2) the fingerprint segmentation method of the MINDTCT module from NBIS [Wa07]; 3) a well known variance-based method presented by Kovesi [Ko]; and 4) the recently proposed factorized directional bandpass (FDB) segmentation method [THG15]. For better readability, the 9 variations of the proposed segmentation framework are denominated according to the feature and clustering algorithm used (i.e. FEATURE_CLUSTERING), as presented in Table 1.

The quantitative evaluation of the proposed methods is made by: (i) a goal-directed performance evaluation (made by replacing the proposed modules in a fingerprint recognition system in order to assess the overall improvement in the system performance); and (ii) a comparison of the automatic orientation and segmentation results with ground-truth data. 
Tab. 1: Nomenclature used to denominate the variations of the proposed segmentation framework.

\begin{tabular}{|l||l||l|}
\hline Extracted feature & Clustering algorithm & Combination nomenclature \\
\hline Range & FCM & RANGE_FCM \\
Entropy & FCM & ENTROPY_FCM \\
Variance & FCM & VARIANCE_FCM \\
Range & k-means & RANGE_kMEANS \\
Entropy & k-means & ENTROPY_kMEANS \\
Variance & k-means & VARIANCE_kMEANS \\
Range & GMM & RANGE_GMM \\
Entropy & GMM & ENTROPY_GMM \\
Variance & GMM & VARIANCE_GMM \\
\hline
\end{tabular}

\subsection{Goal directed evaluation}

Table 2 summarizes the results in all the 19520 images of the FVC databases in terms of $E E R$ for the goal-directed performance evaluation, where the 9 variations of the proposed framework are compared with five state-of-the-art methods by replacing the segmentation module of the $R F V$ system.

It is possible to observe that the $R F V$ achieves, in general, better results when it uses the proposed RANGE_FCM combination as segmentation method. Another observation is that while the range feature is clearly the most robust one, the entropy is not suitable for fingerprint segmentation due to its noisy background sensitivity, specially in low quality fingerprint databases (e.g, FVC2000 DB3, FVC2004 DB3). More concretely, the performance of the fingerprint verification system is improved in 14 of the 16 FVC databases when the segmentation method integrated in the system is one of proposed framework and, in particular, in 9 of the 11 the method is the RANGE_FCM.

In terms of average values, the proposed RANGE_FCM segmentation method promotes the lowest average EER (3.76\%) and the lowest standard deviation (3.55\%). These results indicate that the proposed segmentation algorithm outperforms the existing methods and can handle better with the sensor interoperability problem. When compared with the other five state-of-the-art algorithms, the RANGE_FCM combination promoted an overall improvement in the system performance.

\subsubsection{Ground-truth based evaluation}

In this experiment 320 images from the four FVC databases were randomly selected including 20 images of each subset, to comprise images acquired with different sensors.

The manual segmentation was performed by three specialists in fingerprint recognition. The segmentation error rate $(E r r)$ used to quantify the boundary differences, between the manual segmentation $(G T)$ and the automatic segmentation $(A S)$, is defined as:

$$
E r r=1-\frac{\#(G T \cap A S)}{\#(G T \cup A S)}
$$


6 Pedro M. Ferreira et al.

Tab. 2: Goal-directed evaluation, regarding fingerprint segmentation, in terms of $E E R(\%)$. Numbers in boldface are the best results.

\begin{tabular}{|c|c|c|c|c|c|c|c|c|}
\hline & \multicolumn{4}{|c|}{ FVC2000 } & \multicolumn{4}{|c|}{ FVC2002 } \\
\hline & DB1 & DB2 & DB3 & DB4 & DB1 & DB2 & DB3 & DB4 \\
\hline \multicolumn{9}{|c|}{ Variations of the proposed segmentation framework } \\
\hline RANGE_FCM & 1.86 & 1.09 & 2.65 & 2.32 & 1.46 & 0.79 & 3.94 & 1.75 \\
\hline ENTROPY_FCM & 3.11 & 1.25 & 6.61 & 2.89 & 1.43 & 0.71 & 4.32 & 1.64 \\
\hline VARIANCE_FCM & 1.96 & 1.27 & 10.18 & 2.79 & 1.41 & 0.75 & 4.42 & 1.75 \\
\hline RANGE_kMEANS & 2.04 & 1.14 & 3.29 & 2.86 & 1.36 & 0.68 & 4.79 & 1.68 \\
\hline ENTROPY_kMEANS & 2.04 & 1.45 & 10.06 & 2.85 & 1.39 & 1.00 & 7.18 & 1.79 \\
\hline VARIANCE_kMEANS & 1.86 & 1.14 & 3.21 & 3.01 & 1.36 & 0.75 & 4.39 & 1.75 \\
\hline RANGE_GMM & 3.86 & 1.36 & 4.46 & 2.85 & 1.36 & 0.96 & 6.18 & 1.79 \\
\hline ENTROPY_GMM & 2.04 & 1.36 & 9.98 & 2.85 & 1.39 & 0.96 & 7.14 & 1.61 \\
\hline VARIANCE_GMM & 9.49 & 1.47 & 8.21 & 2.89 & 1.39 & 1.00 & 5.85 & 1.75 \\
\hline \multicolumn{9}{|c|}{ State-of-the-art segmentation algorithms } \\
\hline Fahmy et al. [FT13] & 4.43 & 1.32 & 3.37 & 2.89 & 1.39 & 0.71 & 4.30 & 1.93 \\
\hline NBIS [Wa07] & 2.04 & 1.54 & 10.14 & 2.86 & 1.32 & 0.95 & 7.14 & 2.18 \\
\hline Kovesi [Ko] & 3.68 & 1.61 & 15.04 & 2.75 & 1.43 & 1.46 & 16.32 & 2.27 \\
\hline FDB [THG15] & 1.93 & 0.97 & 2.82 & 2.79 & 1.54 & 0.82 & 3.71 & 1.86 \\
\hline \multirow[t]{3}{*}{ Fingernet [Ta17] } & 3.21 & 2.36 & 6.55 & 4.04 & 7.50 & 3.29 & 10.67 & 4.87 \\
\hline & \multicolumn{4}{|c|}{ FVC2004 } & \multicolumn{4}{|c|}{ FVC2006 } \\
\hline & DB1 & DB2 & DB3 & DB4 & DB1 & DB2 & DB3 & DB4 \\
\hline \multicolumn{9}{|c|}{ Variations of the proposed segmentation framework } \\
\hline RANGE_FCM & 4.93 & 4.46 & 4.87 & 3.61 & 15.60 & 0.60 & 5.23 & 4.99 \\
\hline ENTROPY_FCM & 5.09 & 4.32 & 5.07 & 3.89 & 16.59 & 0.64 & 5.67 & 4.58 \\
\hline VARIANCE_FCM & 4.95 & 4.36 & 5.07 & 3.82 & 16.58 & 0.67 & 5.81 & 4.96 \\
\hline RANGE_kMEANS & 4.93 & 4.29 & 5.00 & 3.96 & 16.55 & 0.68 & 5.88 & 4.81 \\
\hline ENTROPY_kMEANS & 4.82 & 6.29 & 6.57 & 4.16 & 16.53 & 0.93 & 7.21 & 3.38 \\
\hline VARIANCE_kMEANS & 4.95 & 4.32 & 5.11 & 3.89 & 16.59 & 0.69 & 5.86 & 5.09 \\
\hline RANGE_GMM & 4.89 & 6.48 & 5.18 & 4.11 & 16.51 & 0.92 & 8.53 & 3.52 \\
\hline ENTROPY_GMM & 5.21 & 6.29 & 8.42 & 3.96 & 16.50 & 0.84 & 8.78 & 3.56 \\
\hline VARIANCE_GMM & 4.82 & 7.75 & 5.46 & 4.25 & 16.55 & 0.97 & 8.55 & 3.45 \\
\hline \multicolumn{9}{|c|}{ State-of-the-art segmentation algorithms } \\
\hline Fahmy et al. [FT13] & 5.29 & 6.79 & 5.13 & 4.54 & 16.23 & 0.75 & 5.75 & 9.27 \\
\hline NBIS [Wa07] & 4.89 & 6.61 & 8.57 & 4.46 & 16.58 & 2.25 & 10.13 & 3.45 \\
\hline Kovesi [Ko] & 5.03 & 12.71 & 5.98 & 5.98 & 16.24 & 3.76 & 8.39 & 9.69 \\
\hline FDB [THG15] & 5.75 & 5.21 & 6.43 & 4.5 & 16.09 & 0.60 & 6.01 & 8.09 \\
\hline Fingernet [Ta17] & 9.75 & 14.54 & 10.32 & 8.53 & 22.65 & 3.27 & 12.07 & 7.02 \\
\hline
\end{tabular}

Err is a measure of the misclassified pixels in the foreground and the background. The measure used to evaluate the methods is the Average Err which corresponds to the average of the value $E r r$ for the three specialists for each segmentation method.

The results obtained for the ground-truth based evaluation are presented in Table 3 . The RANGE_ kMEANS and RANGE_FCM combinations provided the best results, with an Err of $10.8 \%$. Moreover, the RANGE feature is clearly the most robust and interoperable one, since it provides the best results apart of the clustering algorithm used. The FCM algorithm leads, in general, to better results than the other two clustering methods $(k$ means and GMM) for the same features. Moreover, the best combinations of the proposed 
Robust Clustering-based Segmentation Methods 7

Tab. 3: Ground-truth evaluation, in terms of $\operatorname{Err}(\%)$. The number in boldface is the best result.

\begin{tabular}{|l||c|}
\hline & Average $\operatorname{Err}(\%)$ \\
\hline \hline RANGE_FCM & $\mathbf{1 0 . 8}$ \\
Fahmy et al. [FT13] & 11.3 \\
NBIS [Wa07] & 17.4 \\
Kovesi [Ko] & 21.8 \\
FDB [THG15] & 11.8 \\
Fingernet [Ta17] & 20.5 \\
\hline
\end{tabular}

segmentation framework (i.e. RANGE_ kMEANS; RANGE_FCM; and RANGE_GMM) outperformed the four state-of-art segmentation methods. Fig. 3 shows the segmentation of some images using the proposed RANGE_FCM combination.
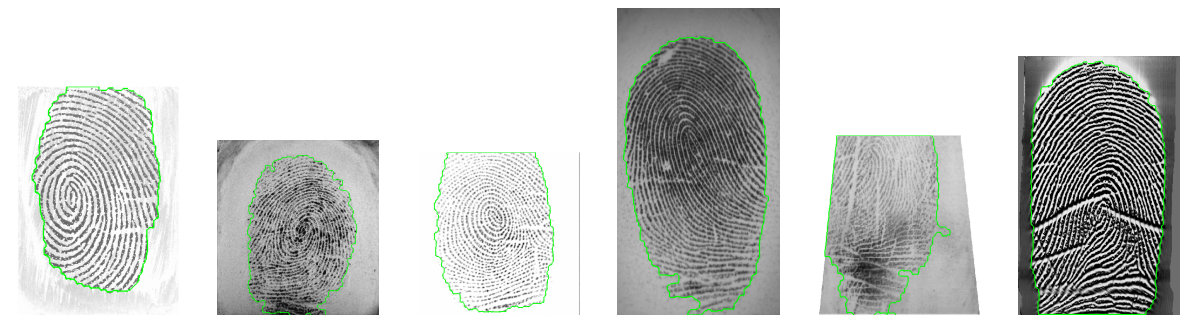

Fig. 3: Results of the RANGE_FCM method in images of the FVC databases (different sensor types).
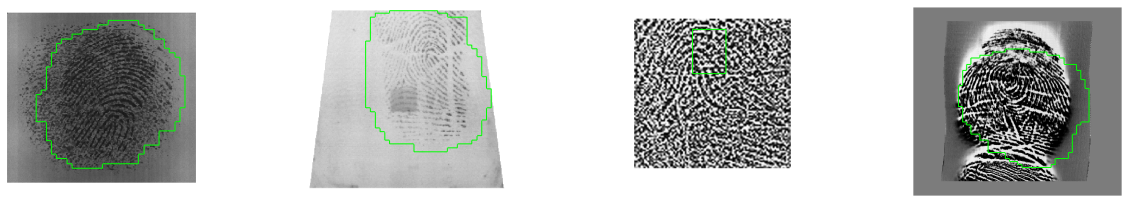

Fig. 4: Results of the Fingernet method in images of the FVC databases (different sensor types).

Impact of the proposed cluster' merging procedure: If the Err is computed by classifying all pixels of the rejected cluster either as foreground or background, taking as baseline the best combination (i.e. RANGE_FCM) the results obtained show an increase of, respectively, $6.62 \%$ and $25.52 \%$ in relation to the Err optimal value of $10.8 \%$. This analysis, clearly demonstrate the importance of the proposed merging procedure in the segmentation process.

\section{Conclusions and future work}

This work proposed a robust binarization process based on clustering algorithms. The method is instantiated by the combination of three different filters: range, entropy and variance filters; and three clustering methods: fuzzy c-means (FCM), k-means and gaussian mixture models (GMM) for pixel classification. The experimental results showed that 
the proposed segmentation method outperforms other existing methods in both segmentation error rate and overall improvement in the fingerprint system recognition performance. In an overall analysis, the ground-truth evaluation showed that the proposed framework for FME leads to improvements in results when compared with the other state-of-the-art methods including a deep learning based method. Regarding the goal directed evaluation, in average the best combination of our proposed method (range filter and fuzzy c-means clustering method) over-perform the other four methods. For future works we foresee as worthwhile testing other feature extraction methods that are even more robust to noise and therefore can lead to better results and comparing more recent deep learning methodologies.

\section{References}

[BG01] Bazen, Asker M.; Gerez, Sabih H.: Segmentation of fingerprint images. In: ProRISC 2001 Workshop on Circuits, Systems and Signal Processing. pp. 276-280, 2001.

[Ch04] Chen, Xinjian; Tian, Jie; Cheng, Jiangang; Yang, Xin: Segmentation of Fingerprint Images Using Linear Classifier. EURASIP J. Appl. Signal Process., pp. 480-494, 2004.

[CKB15] Condessa, Filipe; Kovacevic, Jelena; Bioucas-Dias, José M.: Performance measures for classification systems with rejection. CoRR, abs/1504.02763, 2015.

[FSR15] Ferreira, Pedro M.; Sequeira, Ana F.; Rebelo, Ana: A Fuzzy C-Means Algorithm for Fingerprint Segmentation. In (Paredes, Roberto; Cardoso, Jaime S.; Pardo, Xosé M. eds): Patt. Recog. and Image An., volume 9117 of LNCS, pp. 245-252. Springer, 2015.

[FT13] Fahmy, M.F.; Thabet, M.A.: A fingerprint segmentation technique based on Morphological processing. In: IEEE Int. Symposium on Sig.Proc. and IT (ISSPIT). Dec 2013.

[Ko] Kovesi, Peter: , MATLAB and Octave Functions for Computer Vision and Image Processing. http://www.csse.uwa.edu.au/ p pk/Research/MatlabFns/\#fingerprints.

[KZ09] Kang, Jiayin; Zhang, Wenjuan: Fingerprint image segmentation using modified fuzzy c-means algorithm. In: 3rd Int.Conf. on Bioinf. and Biom. Eng. IEEE, pp. 1-4, 2009.

[Li11] Liu, Eryun; Zhao, Heng; Guo, Fangfei; Liang, Jimin; Tian, Jie: Fingerprint segmentation based on an AdaBoost classifier. Frontiers of Comp. Sc. in China, 5(2):148-157, 2011.

[Ma09] Maltoni, D.; Maio, D.; Jain, A. K.; Prabhakar, S.: Handbook of Fingerprint Recognition. Springer Professional Computing, 2 edition, 2009.

[NCKJ18] Nguyen, Dinh-Luan; Cao, Kai; K. Jain, Anil: , Robust Minutiae Extractor: Integrating Deep Networks and Fingerprint Domain Knowledge, 022018.

[NM14] Nimkar, Rohan; Mishra, Agya: Fingerprint Segmentation Algorithms: A Literature Review. International Journal of Computer Applications, 95(5):20-24, 2014.

[Ra12] Raghotham, Reddy G; Yugander, P; Sheshagiri, Babu J; Rameshwar, Rao R: Possibilistic fuzzy c-means algorithm for fingerprint image. In: 2012 International Conference on Devices, Circuits and Systems (ICDCS). IEEE, pp. 249-253, 2012.

[SC10] Sousa, Ricardo Gamelas; Cardoso, Jaime S.: The Data Replication Method for the Classification with Reject Option. CoRR, abs/1011.3177, 2010. 
[So14] Sousa, Ricardo; da Rocha Neto, Ajalmar R.; Cardoso, Jaime S.; Barreto, Guilherme A.: Classification with Reject Option Using the Self-Organizing Map. In (Wermter, Stefan et al., ed.): Artificial NN and ML - ICANN 2014. Springer, Cham, pp. 105-112, 2014.

[Ta17] Tang, Yao; Gao, Fei; Feng, Jufu; Liu, Yuhang: FingerNet: An unified deep network for fingerprint minutiae extraction. IEEE IJCB, pp. 108-116, 2017.

[THG15] Thai, Duy Hoang; Huckemann, Stephan; Gottschlich, Carsten: Filter Design and Performance Evaluation for Fingerprint Image Segmentation. CoRR, abs/1501.02113, 2015.

[Wa07] Watson, Craig I.; Garris, Michael D.; Tabassi, Elham; Wilson, Charles L.; Mccabe, R. Michael; Janet, Stanley; Ko, Kenneth: , User's Guide NIST Biom. Im. Soft., 2007.

[WTG07] Wu, Chaohong; Tulyakov, Sergey; Govindaraju, Venu: Robust Point-Based Feature Fingerprint Segmentation Algorithm. In (Lee, Seong-Whan; Li, StanZ., eds): Advances in Biometrics, volume 4642 of LNCS, pp. 1095-1103. Springer Berlin Heidelberg, 2007. 